\title{
Analysis of Capacitively Fed Circular Patch Antennas
}

\author{
Zhongbao Wang ${ }^{1,2, a}$, Xiaofeng $\mathrm{Li}^{1, \mathrm{~b}}$, Shaojun Fang ${ }^{1, \mathrm{c}}$ \\ ${ }^{1}$ School of Information Science and Technology, Dalian Maritime University, Dalian, Liaoning \\ 116026, China
}
${ }^{2}$ School of Electronic Engineering, Beijing University of Posts and Telecommunications, Beijing 100876, China

aemail: wangzb@dlmu.edu.cn, bemail: laf900718@sina.com, email: fangshj@dlmu.edu.cn

\section{Keywords: Capacitively fed; Circular patch antenna; Resonant frequency}

\begin{abstract}
In this paper, resonant frequency of capacitively fed circular patch antennas with the various air layer heights and the substrate electrically thickness range from 0.026 to 0.12 is analyzed with HFSS. And the variation of impedance bandwidth is given for capacitively fed circular patch antennas with the substrate electrically thickness increases. The resonant frequency of circular patch antenna with and without capacitor patch is compared. It is shown that there is a good agreement between the theoretical and simulated results.
\end{abstract}

\section{Introduction}

Microstrip patch antennas which have simple structure, light weight and low cost are widely used in communication field. However, as is well known, the impedance bandwidth of the microstrip patch antenna is so narrow. The most common method which broadens the impedance bandwidth is to increase the thickness of the dielectric substrate.

Coaxial probe can be easily fed to any location on the microstrip patch antenna, so it is commonly used in microstrip patch antennas. But with the increase of the substrate thickness, length of the coaxial probe also can increase, which can inevitably lead to an increase for the inductance of the coaxial probe. Therefore, microstrip patch antennas can be the unavoidable mismatch, and the measured resonant frequency is inaccurate. One of solutions to this problem is to cancel out the coaxial probe inductance, which can add a capacitive feed patch [1].

The capacitively fed circular patch antenna is a relatively simple antenna in the practical application, but there is rarely studied by researchers. Simultaneously, the resonant frequency is a vital parameter of the circular patch antenna. Furthermore, there is confusion about the methods to obtain the resonant frequency. Some researchers regard the point of the minimum magnitude of the reflection coefficient as the resonant frequency [2]; other researchers think that the reactance of the input impedance is zero for resonant frequency point [3]. This point is close to the frequency where the resistance reaches a maximum when without regard to the feed structures. However, when the feed structures are included in the analysis procedure for the probe-fed or capacitively-fed patch antennas with thick substrates, above mentioned methods are inaccurate due to the inductance effect of the feed structures. Therefore, the resonant frequency should correspond to a maximum of the resistance and is independent of the reactance [3].

In this work, the goal is to analyze the resonant frequency where the resistance is at a maximum and corresponds to zero reactance for the capacitively fed circular patch antenna. The basic tool employs electromagnetic simulation software ANSYS HFSS.

\section{Structure of Analysis}

In the previous study, there are two main structures. One is a multi-layer capacitively fed patch antenna [1]. A small capacitor patch is embedded in between the radiating patch and the ground plane and dielectric layers are between radiating and capacitor patch and between capacitor patch and the ground plane. This configuration compensates the inductive effect caused by the coaxial 
probe, through feeding the capacitor patch to achieve the feeding radiating patch. But it is difficult to be produced in practice. The other is a single-layer capacitively fed patch antenna [4]. A small capacitor patch is put on the same layer as the radiating patch. The feeding probe is positioned in the center of the small capacitor patch. Both the radiating and capacitor patch are supported by a dielectric substrate which is suspended in air. But this structure will increase the overall size of the antenna.

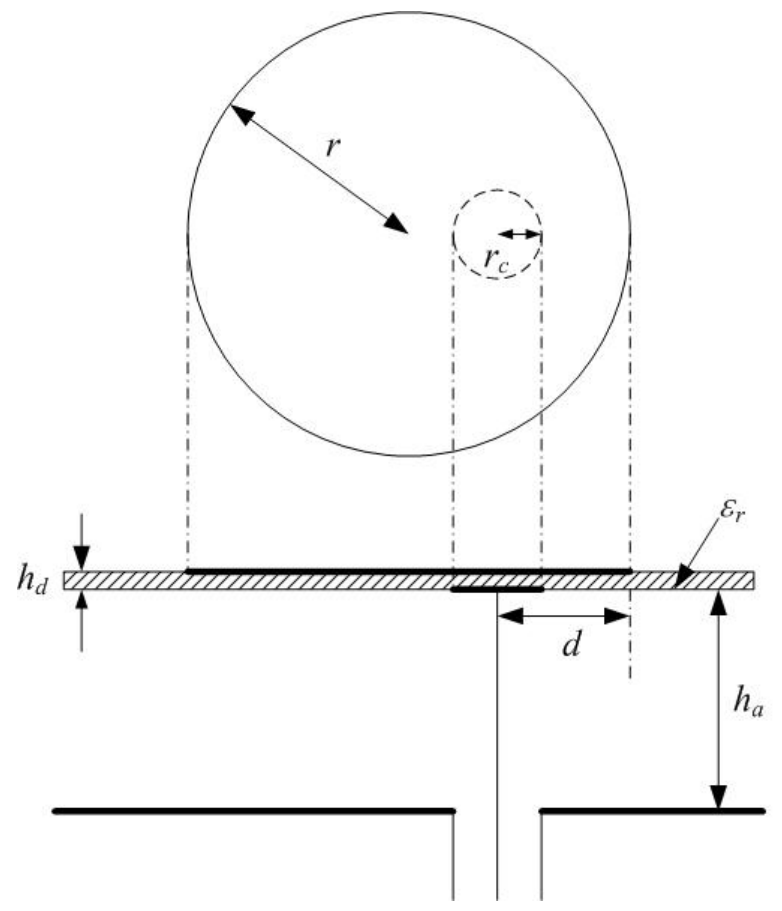

Fig.1. Geometry of capacitively fed circular patch antenna

In this study, the geometry of a capacitively fed circular patch antenna is illustrated in Fig. 1 . The radiating and capacitor patch are respectively in front and back of the dielectric substrate. The feeding coaxial probe is placed in the center of the capacitor patch, so that the radiating patch can be fed. Padding between the dielectric substrate and ground plane is the air.

In this figure, $r$ is the radius of circular patch antenna, $r_{c}$ is the radius of capacitor patch, $d$ is the distance between center of capacitor patch and edge of the circular patch antenna, $h_{d}$ is the thickness of the substrate with dielectric constant $\varepsilon_{r}$ and $h_{a}$ is the height of the air layer. In order to consider the impact of the air layer of the circular patch antenna, equivalent relative dielectric constant is introduced. The dielectric substrate with the thickness $h_{d}$ and the air layer with the height $h_{a}$ are equivalent to a uniform substrate with the thickness $h\left(h_{d}+h_{a}\right)$. Then relative dielectric constant of the uniform substrate is called equivalent relative dielectric constant. Through comparing many literatures, the formula given by literature [5] is relatively accurate. After finishing, the calculation of the equivalent relative dielectric constant is following as:

$$
\begin{gathered}
\varepsilon_{e}^{\prime}=1+\varepsilon_{r} \frac{\left(q_{1}+q_{2}\right)^{2}}{\varepsilon_{r} q_{1}+q_{2}}-q_{1}-q_{2} \\
A=\left(1+\frac{12 h}{r}\right)^{-1 / 2} \\
\varepsilon_{e q}=\frac{2 \varepsilon_{e}^{\prime}-1+A}{1+A}
\end{gathered}
$$

Where $q_{1}$ and $q_{2}$ are padding factors, the expression is written as:

$$
q_{1}=\frac{h_{a}}{2 h}\left\{1+\frac{\pi}{4}-\frac{h}{r_{e w}} \ln \left[\frac{2 r_{e w}}{h_{a}} \sin \left(\frac{\pi h_{a}}{2 h}\right)+\cos \left(\frac{\pi h_{a}}{2 h}\right)\right]\right\}
$$




$$
\begin{gathered}
q_{2}=1-q_{1}-\frac{h}{2 r_{e w}} \ln \left(\frac{\pi r_{e w}}{h}-1\right) \\
r_{e w}=r+\frac{2 h}{\pi} \ln \left[17.08\left(\frac{r}{2 h}+0.92\right)\right]
\end{gathered}
$$

Using (1) to (6), equivalent relative dielectric constant can be obtained. After that, some parameters such as the wavelength in the dielectric substrate $\lambda_{d}$ and electrical thickness of the dielectric substrate $h / \lambda_{d}$ will be calculated by replacing $\varepsilon_{r}$ with $\varepsilon_{e q}$.

$$
\frac{h}{\lambda_{d}}=\frac{h f_{c a l} \sqrt{\varepsilon_{e q}}}{c}
$$

Where $c$ is the velocity of electromagnetic waves in free space and $f_{c a l}$ is the resonant frequency of the circular patch antenna and can be calculated by [6].

Tab.1. Comparison of Resonant Frequencies of Circular Patch Antennas with and without Capacitor Patch

\begin{tabular}{|c|c|c|c|c|c|c|c|}
\hline \multicolumn{3}{|c|}{ Physical and Electrical Parameters } & \multicolumn{3}{c|}{ Resonant frequency $(\mathrm{GHz})$} & $R B W$ \\
\cline { 2 - 8 }$(\mathrm{mm})$ & $h_{a}(\mathrm{~mm})$ & $\varepsilon_{r}$ & $h / \lambda_{d}\left(\times 10^{-2}\right)$ & $f_{\text {cal }}$ & $f_{\text {lump }}$ & $f_{\text {wave }}$ & $(\%)$ \\
\hline 26 & 1 & 7.50 & 2.651 & 2.089 & 2.065 & 2.032 & 1.69 \\
\hline 74 & 6 & 8.00 & 2.718 & 0.936 & 0.927 & 0.915 & 2.75 \\
\hline 142 & 22 & 7.00 & 4.241 & 0.501 & 0.500 & 0.494 & 5.55 \\
\hline 102 & 20 & 7.00 & 5.282 & 0.679 & 0.667 & 0.656 & 7.16 \\
\hline 66 & 16 & 2.00 & 6.439 & 1.050 & 1.052 & 1.033 & 11.18 \\
\hline 86 & 25 & 4.40 & 7.370 & 0.782 & 0.771 & 0.762 & 14.55 \\
\hline 100 & 31 & 2.55 & 7.690 & 0.677 & 0.678 & 0.668 & 16.44 \\
\hline 85 & 30 & 2.00 & 8.597 & 0.788 & 0.789 & 0.774 & 20.09 \\
\hline 117 & 52 & 6.50 & 10.318 & 0.547 & 0.547 & 0.533 & 22.09 \\
\hline 88 & 44 & 2.55 & 11.396 & 0.722 & 0.719 & 0.704 & 24.37 \\
\hline 52 & 27 & 1.50 & 11.919 & 1.223 & 1.222 & 1.209 & 26.49 \\
\hline 55 & 32 & 2.55 & 13.054 & 1.118 & 1.105 & - & 20.00 \\
\hline 130 & 80 & 3.30 & 13.351 & 0.472 & 0.472 & - & 21.10 \\
\hline \multicolumn{3}{|c|}{ Average relative error (\%) } & 0.585 & 1.618 & - \\
\hline \multicolumn{3}{|c|}{ Max relative error (\%) } & 1.767 & 2.559 & - \\
\hline
\end{tabular}

\section{Results and Discussion}

To appreciate the validity and accuracy of the method to obtained resonant frequency, simulated resonant frequencies of two models for electrically thin and thick circular patch antennas with different physical dimensions with various substrates are compared. One is the circular patch antenna fed without capacitor patch through lumped port excitation. Another is the circular patch antenna fed with capacitor patch through wave port excitation. In two models, thickness of the dielectric substrate is $1.5 \mathrm{~mm}$. Then change of air layer height $h_{a}$ affects the substrate electrically thickness $h / \lambda_{d}$. The results are shown in Table $1 . f_{\text {cal }}$ is the resonant frequency calculated by literature [6]. $f_{\text {lump }}$ and $f_{\text {wave }}$ are the resonant frequencies obtained by lumped port excitation to the circular patch antenna without capacitor patch and wave port excitation to the circular patch antenna with capacitor patch. In the lumped port excitation, for convenience, it is at half of the radius for the circular patch antenna because the feeding position has little effect on the resonant frequency. In wave port excitation, through adjusting feeding position and the radius of capacitor patch, resistance and reactance of the input impedance are respectively close to 50 and 0 Ohms. The resonant frequency $f_{\text {lump }}$ and $f_{\text {wave }}$ are both the point where resistance is at a maximum. $R B W$ is the relative bandwidth of capacitively fed circular patch antenna, which is $10 \mathrm{~dB}$ bandwidth of return loss 
divided by the resonant frequency $f_{\text {wave }}$.

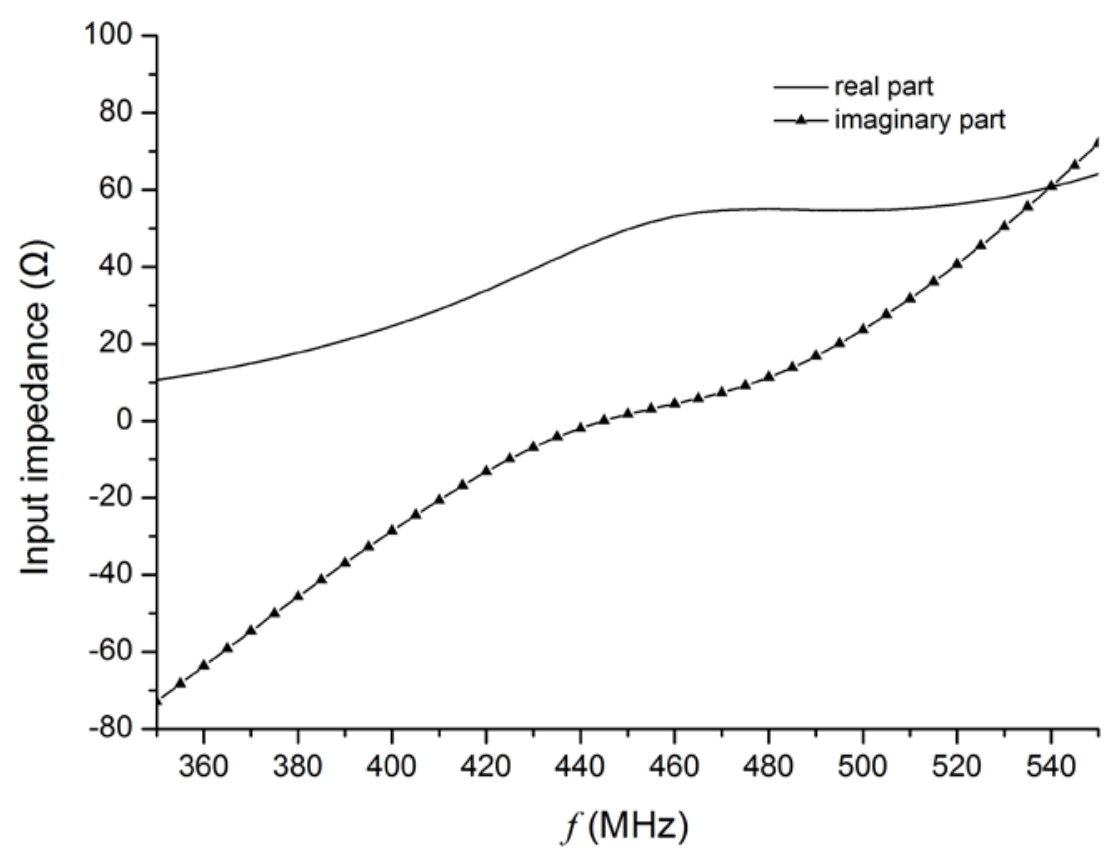

(a) Input impedance

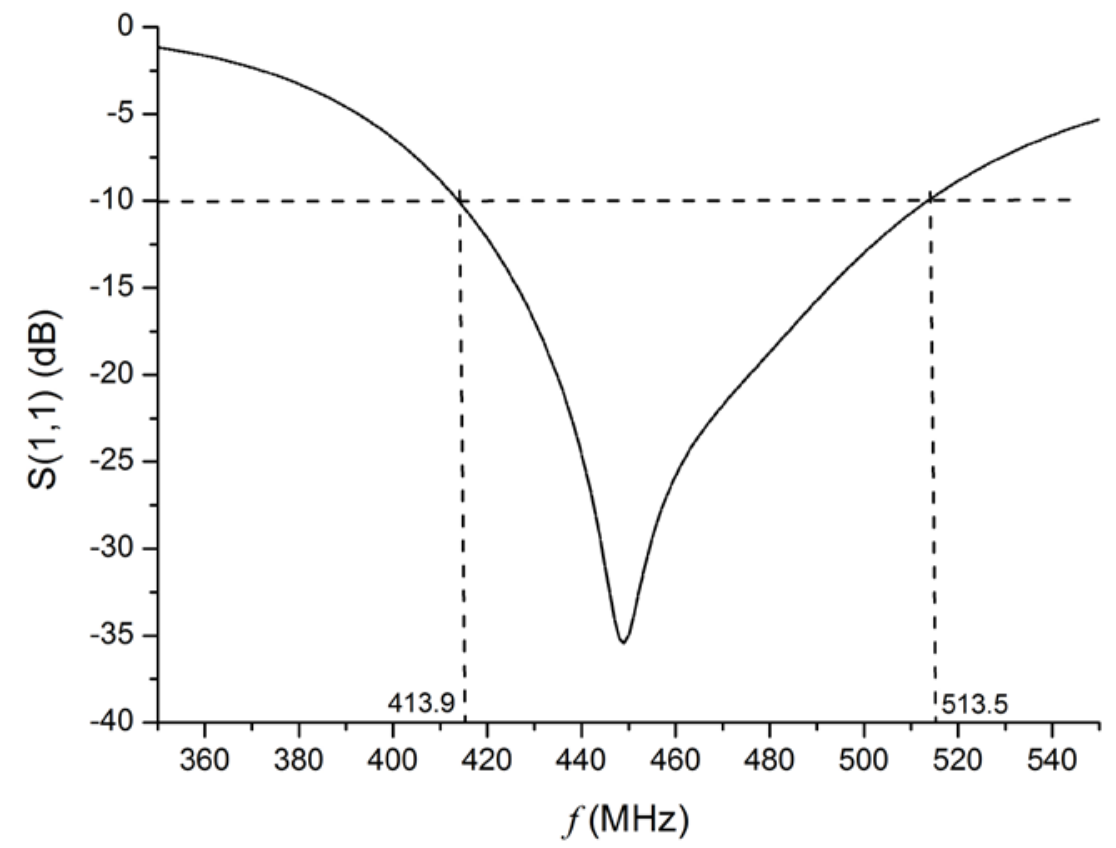

(b) $S$-parameters

Fig.2. Input impedance and $S$-parameters of capacitively fed circular patch antenna

$$
\text { ( } r=130 \mathrm{~mm}, h_{a}=80 \mathrm{~mm}, \varepsilon_{r}=3.3 \text { ) }
$$

It is observed from Table 1 that the average relative error is less than $1 \%$ with max relative error less than $2 \%$ between $f_{\text {lump }}$ and $f_{\text {cal }}$. It shows that equivalent relative dielectric constant is accurate to calculate the resonant frequency and $f_{\text {lump }}$ is reliable with HFSS simulation. Further, $f_{\text {wave }}$ is very close to $f_{\text {lump }}$ for the different air layer height $h_{a}$ with a variety of dielectric constant. The average relative error is less than $2 \%$ and max relative error is less than $3 \%$. In addition, with substrate 
electrically thickness increases, $R B W$ is gradually broadened. This shows that capacitively fed circular patch antenna with electrical thick substrate can broaden bandwidth. In the last row of Table 1 as an example, the results are shown in Fig. 2 when the substrate electrically thickness $h / \lambda_{d}$ is more than 0.12. It is clear from Fig. 2(a) that the curve of input impedance has been distorted. The resonant frequency $f_{\text {wave }}$ can't be obtained. In Fig. 2(b), $413.9 \mathrm{MHz}$ and $513.5 \mathrm{MHz}$ are points where the return loss is $-10 \mathrm{~dB}$. $R B W$ is calculated with replacing $f_{\text {wave }}$ with $f_{\text {lump}}, R B W=$ (513.5-413.9)/472=21.10\%, which won't increase. Obviously, it does not make sense to study on capacitively fed circular patch antenna with substrate electrically thickness more than 0.12. Moreover, if the substrate electrically thickness is too thin, the reactance brought by the coaxial probe has little influence. Thence, there is no analysis capacitively fed circular patch antenna of which substrate electrically thickness $h / \lambda_{d}$ is less than 0.026 .

\section{Conclusion}

In this paper, the resonant frequency of capacitively fed circular patch antennas is analyzed. It is indicated that the resonant frequency obtained for capacitively fed circular patch antennas which vary in substrate electrically thickness $h / \lambda_{d}$ from 0.026 to 0.12 with various air layer $h_{a}$ is accurate when resistance is at a maximum. Except for, increase of substrate electrically thickness $h / \lambda_{d}$ which is less than 0.12 and more than 0.026 can broaden the bandwidth. Also, a significant analysis can be used for many engineering applications.

\section{Acknowledgement}

This work was supported jointly by the National Natural Science Foundation of China (nos. 61401056 and 61571075), the Doctor Startup Foundation of Liaoning Province (no. 20141103), the Scientific Research Project of the Department of Education of Liaoning Province (no. L2013196), the China Postdoctoral Science Foundation (no. 2015M580070), and the Fundamental Research Funds for the Central Universities (no. 3132015212).

\section{References}

[1] G.A.E. Vandenbosch and A.R. Van de Capelle, "Study of the capacitively fed microstrip antenna element,” IEEE Trans Antennas Propagat. vol. 42, pp. 1648-1652, December 1994.

[2] M. Kara, "Closed-form expressions for the resonant frequency of rectangular microstrip antenna elements with thick substrates,” Microwave Opt Technol Lett. vol. 12, pp. 131-136, June 1996.

[3] E. Chang, S.A. Long and W.F. Richards, "An experimental investigation of electrically thick rectangular microstrip antennas,” IEEE Trans Antennas Propagat. vol. 34, pp. 767-772, June 1986.

[4] G. Mayhew-Ridgers, J.W. Odendaal and J. Joubert, "Single-layer capacitive feed for wideband probe-fed microstrip antenna elements,” IEEE Trans Antennas Propagat. vol. 51, pp. 1405-1407, June 2003.

[5] S.S. Zhong, G. Liu and G. Qasim, "Closed form expressions for resonant frequency of rectangular patch antennas with multidielectric layers,” IEEE Trans Antennas Propagat. vol. 42, pp. 1360-11363, September 1994.

[6] T. Günel, "Continuous hybrid approach to the modified resonant frequency calculation for circular microstrip antennas with and without air gaps,” Microwave Opt Technol Lett. vol. 40, pp. 423-427, March 2004. 